\title{
SapC-DOPS nanovesicles induce Smac- and Bax-dependent apoptosis through mitochondrial activation in neuroblastomas
}

\author{
Mahaboob K Sulaiman', Zhengtao Chu ${ }^{1,2}$, Victor M Blanco', Subrahmanya D Vallabhapurapu', \\ Robert S Franco ${ }^{1}$ and Xiaoyang $\mathrm{Qi}^{1,2^{*}}$
}

\begin{abstract}
Background: High toxicity, morbidity and secondary malignancy render chemotherapy of neuroblastoma inefficient, prompting the search for novel compounds. Nanovesicles offer great promise in imaging and treatment of cancer. SapC-DOPS, a stable nanovesicle formed from the lysosomal protein saposin C and dioleoylphosphatidylserine possess strong affinity for abundantly exposed surface phosphatidylserine on cancer cells. Here, we show that SapC-DOPS effectively targets and suppresses neuroblastoma growth and elucidate the molecular mechanism of SapC-DOPS action in neuroblastoma in vitro.
\end{abstract}

Methods: In vivo targeting of neuroblastoma was assessed in xenograft mice injected intravenously with fluorescentlylabeled SapC-DOPS. Xenografted tumors were also used to demonstrate its therapeutic efficacy. Apoptosis induction in vivo was evaluated in tumor sections using the TUNEL assay. The mechanisms underlying the induction of apoptosis by SapC-DOPS were addressed through measurements of cell viability, mitochondrial membrane potential $(\Delta \Psi \mathrm{M})$, flow cytometric DNA fragmentation assays and by immunoblot analysis of second mitochondria-derived activator of caspases (Smac), Bax, Cytochrome c (Cyto c) and Caspase-3 in the cytosol or in mitochondrial fractions of cultured neuroblastoma cells.

Results: SapC-DOPS showed specific targeting and prevented the growth of human neuroblastoma xenografts in mice. In neuroblastoma cells in vitro, apoptosis occurred via a series of steps that included: (1) loss of $\Delta \psi M$ and increased mitochondrial superoxide formation; (2) cytosolic release of Smac, Cyto c, AIF; and (3) mitochondrial translocation and polymerization of Bax. ShRNA-mediated Smac knockdown and V5 peptide-mediated Bax inhibition decreased cytosolic Smac and Cyto c release along with caspase activation and abrogated apoptosis, indicating that Smac and Bax are critical mediators of SapC-DOPS action. Similarly, pretreatment with the mitochondria-stabilizing agent bongkrekic acid decreased apoptosis indicating that loss of $\triangle \Psi \mathrm{M}$ is critical for SapC-DOPS activity. Apoptosis induction was not critically dependent on reactive oxygen species (ROS) production and Cyclophilin D, since pretreatment with $\mathrm{N}$-acetyl cysteine and cyclosporine A, respectively, did not prevent Smac or Cyto c release.

Conclusions: Taken together, our results indicate that SapC-DOPS acts through a mitochondria-mediated pathway accompanied by an early release of Smac and Bax. Specific tumor-targeting capacity and anticancer efficacy of SapC-DOPS supports its potential as a dual imaging and therapeutic agent in neuroblastoma therapy.

Keywords: SapC-DOPS, Saposin C, Dioleoylphosphatidylserine, Mitochondria-mediated apoptosis, Smac/Diablo, Bax polymerization

\footnotetext{
*Correspondence: xiaoyang.qi@uc.edu

${ }^{1}$ Division of Hematology and Oncology, Department of Internal Medicine,

University of Cincinnati College of Medicine, Cincinnati, Ohio, USA

2Divison of Human Genetics, Department of Pediatrics, Cincinnati Children's

Hospital Medical Center, Cincinnati, Ohio, USA
}

\section{Ciomed Central}

(c) 2015 Sulaiman et al.; licensee BioMed Central. This is an Open Access article distributed under the terms of the Creative Commons Attribution License (http://creativecommons.org/licenses/by/4.0), which permits unrestricted use, distribution, and reproduction in any medium, provided the original work is properly credited. The Creative Commons Public Domain Dedication waiver (http://creativecommons.org/publicdomain/zero/1.0/) applies to the data made available in this article, unless otherwise stated. 


\section{Introduction}

Nanotechnology is transforming cancer therapy by improving drug delivery, imaging and selective targeting of tumor cells [1-3]. In the past, several attempts have been made to formulate nanovesicles from components normally present in cells [4]. In this direction, SapC-DOPS, a nanovesicle made from saposin $\mathrm{C}$ and dioleylphosphatidylserine, is a unique protein-lipid complex that selectively targets and kills human cancer cells in vitro and in vivo [1,5-12]. Saposin C is an $80 \mathrm{kDa}$ heat-stable, proteaseresistant protein containing distinct functional domains; that functions as a co-activator of sphingolipid-degrading lysosomal hydrolases (sphingomyelinase and acid $\beta$ glucosidase) [13]. Owing to the presence of a fusogenic domain comprising two amphipathic $\alpha$-helices containing four Lys residues (K13, K17, K26, and K38), SapC exhibits natural affinity towards negatively charged phospholipids such as phosphatidylserine (PS). This interaction occurs at low $\mathrm{pH}$ (pKa of 5.3) and is critical for SapC activation [13,14]. We have previously assembled SapC and DOPS into stable nanovesicles and its efficacy and safety profiles have been established in various forms of cancer $[1,5,6,8,12]$. SapC-DOPS is hypothesized to bind to exposed PS on the cancer cell surface and induce apoptosis by increasing intracellular ceramide level leading to subsequent caspase activation [8]. However, the precise intracellular pathway(s) mediating SapC-DOPS induced apoptotic cancer cell death is still unknown.

Neuroblastoma accounts for $15 \%$ of all pediatric cancer mortalities and is the most common extracranial tumor in young adults [15]. Aggressive chemotherapy and radiation protocols have failed to improve the survival rates significantly in children with high-risk disease [16]. Efficacy of chemotherapy in neuroblastoma is less than satisfactory due to several factors such as high toxicity, severe morbidity and risk of secondary malignancy [17]. Moreover, in patients with relapse the long-term survival rates are $<50 \%$ emphasizing the need for novel, non-genotoxic targeted therapies. Elevation of intracellular ceramide, a known regulator of mitochondrial function, was noticed during in vitro treatment of neuroblastoma cell lines with SapC-DOPS [8]. Ceramide induces apoptosis via the mitochondrial pathway [18]. Mitochondria play a central role in the induction of apoptosis by acting as both a major amplification step and the principal site of action for pro- and anti-apoptotic members of the $\mathrm{Bcl}-2$ family [19]. Whereas the anti-apoptotic members (e.g.,Bcl-2 and $\mathrm{Bcl}-\mathrm{xL}$ ) confine apoptogenic proteins within the mitochondrial intermembrane space by promoting pore closure, the pro-apoptotic proteins (e.g., Bax, Bak and Bid) that translocate from the cytosol to mitochondria promote pore opening [20]. In addition, it is known that these pro-apoptotic molecules promote pore formation independently or in combination with other mitochondrial proteins such as the voltage-dependent anion channel (VDAC) effecting the release of apoptogenic proteins such as Cyto c, Smac/Diablo and apoptosis inducing factor (AIF) from the intermembrane space [21-23]. Final commitment to apoptosis is postulated to occur by the following sequence of events: formation of pores or channels in the outer mitochondrial membrane, opening of pores, loss of mitochondrial membrane potential $(\triangle \Psi \mathrm{M})$, apoptogenic protein release from mitochondria and caspase activation [24]. In this report we evaluate the in vivo targeting and antitumor capacity of SapC-DOPS in mice bearing neuroblastoma xenografts, and address the molecular mechanisms underlying neuroblastoma cell death after SapC-DOPS exposure. Based on past observations, we test the hypothesis that SapC-DOPS-induced apoptotic cell death is caused by mitochondrial dysfunction, apoptogenic protein release and caspase activation. Cell viability, mitochondrial function and redistribution of apoptotic proteins are assessed in vitro in the neuroblastoma cell lines SK-N-SH and IMR-32, representative of nonmetastatic and metastatic neuroblastoma, respectively. The in vivo efficacy of SapC-DOPS in suppressing neuroblastoma growth and the insights obtained into its mechanism of action support its potential as a novel therapeutic agent for the treatment of neuroblastoma.

\section{Materials and methods \\ Reagents and antibodies}

The following reagents and antibodies were used: Bongkrekic acid (Santa Cruz Biotechnology, La Jolla, CA), JC-1 (eBioscience, San Diego, CA), Cyclosporine A, $2^{\prime}, 7^{\prime}$-dichlorofluorescein acetate (DCFH-DA), N-acetyl cysteine (Sigma, St.Louis, MO), Dioleylphosphatidylserine (Avanti Lipids, Alabaster, AL), 3-(4,5-dimethylthiazol-2yl)-2,5-diphenyltetrazolium bromide (MTT) ((Roche Diagnostics, Indianapolis, IN), and disuccinyl suberate (Thermo Scientific Fischer, Rockford, IL). Anti-Bcl-2, anti- $\beta$-Actin (Abcam, Cambridge, MA), anti-Cyto c (eBioscience, San Diego, CA), anti-AIF, anti-caspase-3, anti-cleaved caspase-3 (Cell Signaling Technology, Boston, MA, anti-Survivin, Smac/Diablo, $\alpha$-Tubulin (Novus biological, Littleton, CO), anti-COX-4, anti-Bax (N-20; Santa Cruz Biotechnology, La Jolla, CA), antiBax (polymer-recognizing A67 clone; Sigma, St.Louis, MO) and anti- cleaved PARP (Millipore, Bedford, MA).

Animal maintenance and experimental procedures were carried out in accordance with the US National Institute of health Guidelines for Use of Experimental Animals and approved by the Institutional Animal Care and Use Committee of the University of Cincinnati and Cincinnati Children's Hospital Medical Center. 


\section{Preparation and characterization of SapC-DOPS nanovesicles}

The procedures for production of SapC-DOPS nanovesicles have been described in detail before [1,6-9]. Briefly, recombinantly expressed and purified saposin $\mathrm{C}$ along with solvent-dried dioleylphosphatidylserine were mixed in acidic citrate-phosphate buffer and freshly assembled into nanovesicles by bath sonication. The lipophilic, infrared dye CellVue Maroon (CVM) was added during SapC-DOPS assembly to fluorescently label the nanovesicles for in vivo imaging of neuroblastoma human xenografts. The nanovesicles are stable for at least a week, when stored at $4^{\circ} \mathrm{C}$. TEM analysis for surface morphology was performed as described earlier [25].

\section{Mouse xenografts and cell culture}

Human neuroblastoma CHLA-20 was a gift from Thomas Inge (Cincinnati Children's Hospital Medical Center); the origin and culture conditions were previously described [26]. Athymic nude mice (nu/nu, NIH) (15 mice per group), were injected with $7.5 \times 10^{6}$ cells subcutaneously to initiate tumor growth. When tumors reached a volume of $400 \mathrm{~mm}^{3}$, five doses of either SapCDOPS (SapC $4 \mathrm{mg} / \mathrm{kg}$ body weight, DOPS $2 \mathrm{mg} / \mathrm{kg}$ body weight) or PBS (control) were intratumorally administered once every 3 days. Tumor growth was assessed periodically with a caliper, and after 16 days, tumors were excised, weighted, and processed for hematoxylin and eosin staining and apoptosis (TUNEL) assays.

The human neuroblastoma SK-N-SH and IMR-32 cell lines were obtained from American Type Culture Collection and grown in AMEM supplemented with 10\% FBS. Human Schwann cells (ScienCell Research Laboratories, Carlsbad, CA) were grown as recommended by the supplier. After overnight attachment, cells were treated with either DOPS or SapC-DOPS for concentration- or timedependence assays. Where indicated, cells were pretreated for $60 \mathrm{~min}$ with bongkrekic acid, cyclosporine A or Nacetyl cysteine.

\section{Cell viability and apoptosis assays}

Cell viability was assessed with a standard assay using the tetrazolium dye MTT (3-(4,5-dimethylthiazol-2-yl)-2,5-diphenyltetrazolium bromide) as previously described [6] three days after initiating treatment. The following methods were employed to assess SapC-DOPS-induced cell death: G6PD release assay (Life Technologies, Grand Island, NY), was performed according to the manufacturer's instructions, DAPI staining was performed as described earlier [27], caspase activation through cleaved caspase- 3 and cleaved PARP fragments was evaluated by Western blotting, and cell cycle analysis was performed on a FACS Calibur (Becton Dickinson) in serum-starved, synchronized cells after fixation with $80 \%$ ethanol at $-20^{\circ} \mathrm{C}$ for
$20 \mathrm{~min}$ followed by staining with $100 \mu \mathrm{g} / \mathrm{ml}$ of RNase and $25 \mu \mathrm{g} / \mathrm{ml}$ of PI [28]. Cell cycle phase was analyzed with the CellQuest-Pro software program (Becton Dickinson). In vivo apoptosis was measured by TUNEL staining as described earlier [8].

\section{Evaluation of mitochondrial membrane potential $(\Delta \Psi \mathrm{M})$ and ROS production}

Following treatment with SapC-DOPS, cells in triplicate were washed with PBS and evaluated for concentrationand time-dependent changes in $\triangle \Psi \mathrm{M}$ by resuspension in fresh JC-1 containing medium, followed by $30 \mathrm{~min}$ incubation in the dark at room temperature [28]. Fluorescence intensity was measured with excitation at $490 \mathrm{~nm}$ and the emission monitored at 530 (monomer) and 590 (aggregate) nm, using a BMG microplate reader (BMG Labtech, Inc., Durham, NC). The ratio between green and red fluorescence provides an estimate of $\triangle \Psi M$ that is independent of the mitochondrial mass. For the ROS assay, cells treated with SapC-DOPS were exposed to DCFH-DA for $15 \mathrm{~min}$ at $37^{\circ} \mathrm{C}$. Fluorescence excitation and emission wavelengths were set at 480 and $530 \mathrm{~nm}$ respectively, using a BMG microplate reader (BMG Labtech, Inc., Durham, NC). Mitochondrial superoxide was detected using the fluorescent Mito-Sox probe (Invitrogen). Cells were incubated in Hank's buffer with $2 \mu \mathrm{M}$ MitoSox-Red for $30 \mathrm{~min}$ at $37^{\circ} \mathrm{C}$ in a $5 \% \mathrm{CO}_{2}$ atmosphere, washed with $\mathrm{PBS}$ and the fluorescence assessed by flow cytometry. Positive control cells were pretreated with $20 \mu \mathrm{M}$ Antimycin A for $20 \mathrm{~min}$ at room temperature. We used the FL1, FL2 and FL3 channels of a FACScalibur flow cytometer $(15 \mathrm{~mW}$ argon ion laser tuned at $488 \mathrm{~nm}$; CellQuest software, Becton Dickinson Biosciences). Thresholds were adjusted by using nonstained and stained cells for MitoSox-Red fluorescence.

\section{Flow cytometric evaluation of $\mathrm{Ca}^{2+}$ by Fluo-3 AM assay}

Intracellular calcium was measured by flow cytometry using the cell permeant, $\mathrm{Ca}^{2+}$-sensitive fluorescent dye Fluo-3 AM (Life technologies, Carlsbad, CA) [29]. After treatment with SapC-DOPS for different time points, cells were washed in serum-free Advanced-MEM media, and incubated with Fluo-3 AM for $30 \mathrm{~min}$ at $37^{\circ} \mathrm{C}$. Later, cells were washed with HEPES (4-(2-hydroxyethyl)-1-piperazineethanesulfonic acid) buffer, trypsinized and centrifuged at 3,500 $\mathrm{rpm}$ for $5 \mathrm{~min}$. The pellet was resuspended in HEPES buffer and analyzed using FACS Calibur (Becton Dickinson) with excitation at $488 \mathrm{~nm}$ and emission at $525 \mathrm{~nm}$. Approximately 10,000 events were counted.

\section{Western blotting}

After treatment with SapC-DOPS, cells were trypsinized and lysed with RIPA buffer (Sigma) containing protease inhibitor (Thermo Pierce) for $30 \mathrm{~min}$ on ice. The lysates 
were centrifuged at $11,300 \mathrm{~g}$ for $20 \mathrm{~min}$ at $4^{\circ} \mathrm{C}$ to collect supernatant. Protein concentration was determined by the BCA method (Thermo Fischer Scientific, Rockford, IL). Equal amounts of proteins $(30-50 \mu \mathrm{g})$ were separated by $4-20 \%$ gradient sodium dodecyl sulphatepolyacrylamide gel electrophoresis (SDS-PAGE) and transferred to PVDF membrane (Amersham). After blocking, the membrane was incubated overnight at $4^{\circ} \mathrm{C}$ with primary antibodies. Following this, blots were probed with the appropriate Li-COR secondary antibodies conjugated with IRDye $800 \mathrm{CW}$ or IRDye 680 LT. Proteins were visualized using an Odyssey IR scanner and quantified using Odyssey software (LI-COR Biosciences, Lincoln, NE).

\section{Mitochondrial and cytosolic fractionation}

The mitochondrial and cytoplasmic fractions were separated using the Mitochondrial/Cytosol fractionation kit (BioVision, CA, USA) as per the manufacturer's instructions. Briefly, whole-cell pellets dissolved in cytosolic fraction extraction buffer were subjected to 55 strokes in a $2 \mathrm{ml}$ Dounce homogenizer on ice. The homogenate was centrifuged at $3,500 \mathrm{rpm}$ for $10 \mathrm{~min}$ at $4^{\circ} \mathrm{C}$ to pellet nuclei and unbroken cells. The supernatant was subsequently centrifuged at $13,000 \mathrm{rpm}$ for $30 \mathrm{~min}$ at $4^{\circ} \mathrm{C}$ to obtain cytosolic supernatant and the mitochondrial pellet. Mitochondrial pellets were resuspended in mitochondrial extraction buffer by gentle vortex for $30 \mathrm{sec}$.

\section{Bax oligomerization and bax inhibition}

Bax oligomerization with cross linking was detected as described previously [27]. Briefly, cells after treatment with SapC-DOPS cells were washed in conjugating buffer followed by cross linking with $2 \mathrm{mM}$ disuccinyl suberate in non-reducing buffer and incubated for $30 \mathrm{~min}$ at room temperature. Reaction was quenched by addition of Tris$\mathrm{HCl}(\mathrm{pH} 7.5)$ and incubation at room temperature for $15 \mathrm{~min}$. The samples were then solubilized in lysis buffer containing Nonidet P-40 without a reducing agent and centrifuged at 12,000 $\mathrm{x} g$ for $10 \mathrm{~min}$. Bax oligomers were detected using the A67 clone that exclusively detects the polymerized form. Bax inhibition was performed as described earlier [30]. Briefly, cells were pre-incubated with Bax inhibiting peptide (V5) or negative control peptide (EMD Millipore,Chicago, IL) for one hour, prior to SapCDOPS treatment.

Lentiviral infection and stable knockdown of Smac/Diablo Permanent knockdown of Smac was achieved by expression of pre-validated shRNA targeting the sequence CCGACAATATACAAGTTTACT in Smac (shSmac) available as clone ID TRCN04513 in the Sigma-TRC consortium database. DNA oligonucleotides encoding for shSmac were annealed and cloned into pLKO.1 puro. Lentivirus particles carrying shSmac were produced by transfecting 293T cells with pLKO.1-puro-shSmac together with viral packaging vectors (psPAX2, pMD2G) by calcium phosphate transfection at the Cincinnati Children's Hospital Medical Center viral vector core facility. Three days post infection of SK-N-SH cells with Smac shRNA containing virus, cells were selected in a medium containing puromycin (Life Technologies, Grand Island, NY). Efficiency of the knockdown was checked by Western blot. Cells transfected with the empty vector served as control.

\section{Statistical analysis}

Data are represented as the mean \pm SE. Statistical analyses were done with the Student's $t$ test and $\mathrm{P}<0.05$ was considered significant.

\section{Results}

SapC-DOPS targets neuroblastoma and inhibits tumor growth in vivo

Saposin C and dioleoylphosphatidylserine were assembled (pH 5.0) at a molar ratio of 1:7 for in vivo studies or 1:3 for in vitro studies. Tumor targeting was evaluated by real-time fluorescent imaging, following an intravenous injection of fluorescently (CVM)-labeled SapC-DOPS nanovesicles (mouse 1), CVM-labeled DOPS (mouse 2), or SapC + CVM (mouse 3) in nude mice bearing human neuroblastoma xenografts produced by flank injections of CHLA-20 cells. At $24 \mathrm{~h}$ post injection, fluorescent signal was only detected in tumor-bearing mouse that received CVM-labeled SapC-DOPS (Figure 1A).

To assess the therapeutic efficacy of SapC-DOPS on neuroblastoma, mice bearing CHLA-20 neuroblastoma xenografts were subjected to intratumoral injections of SapC-DOPS $(\mathrm{SapC}=4 \mathrm{mg} / \mathrm{kg}, \quad \mathrm{DOPS}=2 \mathrm{mg} / \mathrm{kg}$ ) or phosphate buffered saline (PBS). Mice treated with SapC-DOPS showed a significant inhibition of tumor growth $(\mathrm{P}=0.0097)$ and decreased tumor weight $(\mathrm{P}<0.0001)$ when compared to mice treated with $\mathrm{PBS}$ (Figure 1B). Histological evaluation by hematoxylineosin $(\mathrm{H} \& \mathrm{E})$ and terminal deoxynucleotidyltransferase dUTP nick end labeling (TUNEL) staining showed extensive induction of apoptosis within neuroblastoma tissue (Figure 1C, D) in the mouse xenografts. Since SapC-DOPS showed specific tumor targeting and induced apoptosis in mouse xenografts, we proceeded to determine the mechanism behind apoptosis induction in established neuroblastoma cell lines.

\section{SapC-DOPS triggers apoptosis of neuroblastoma cells}

We hypothesized that the characteristically acidic tumor microenvironment facilitates the binding of SapC-DOPS to exposed PS on tumor cells, activates lysosomal glucosylceramide breakdown, and elevates ceramide levels leading to apoptotic cell death [6]. Although elevated 


\section{A CVM fluorescence}

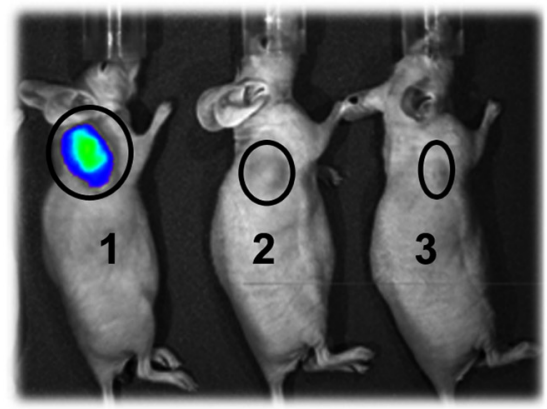

Photograph

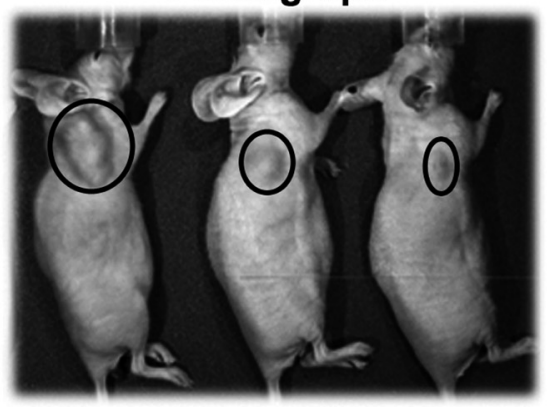

C
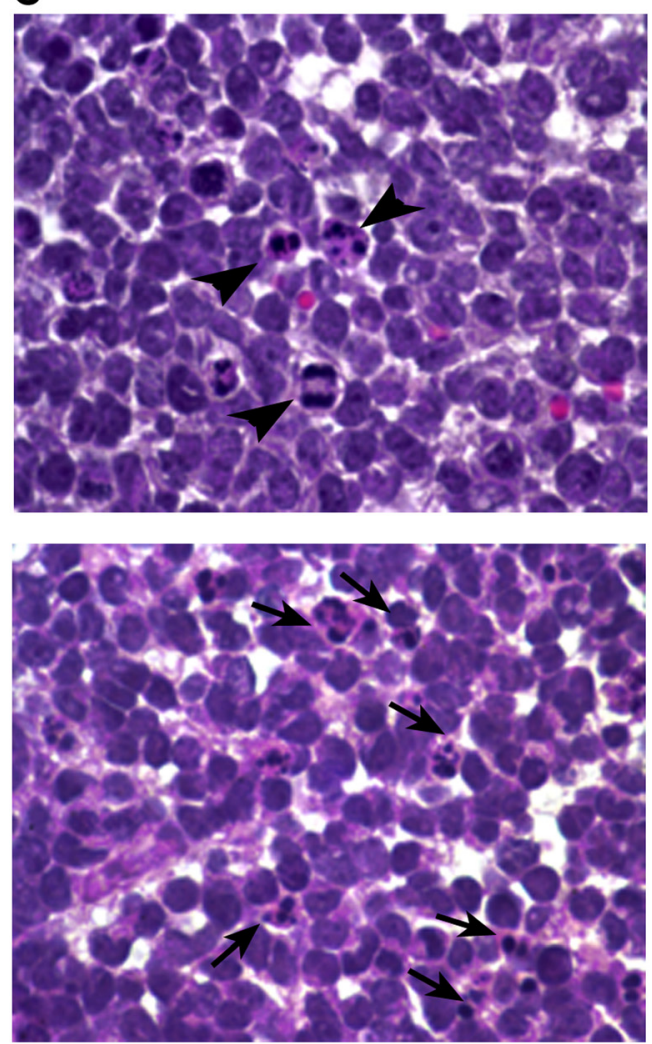

B
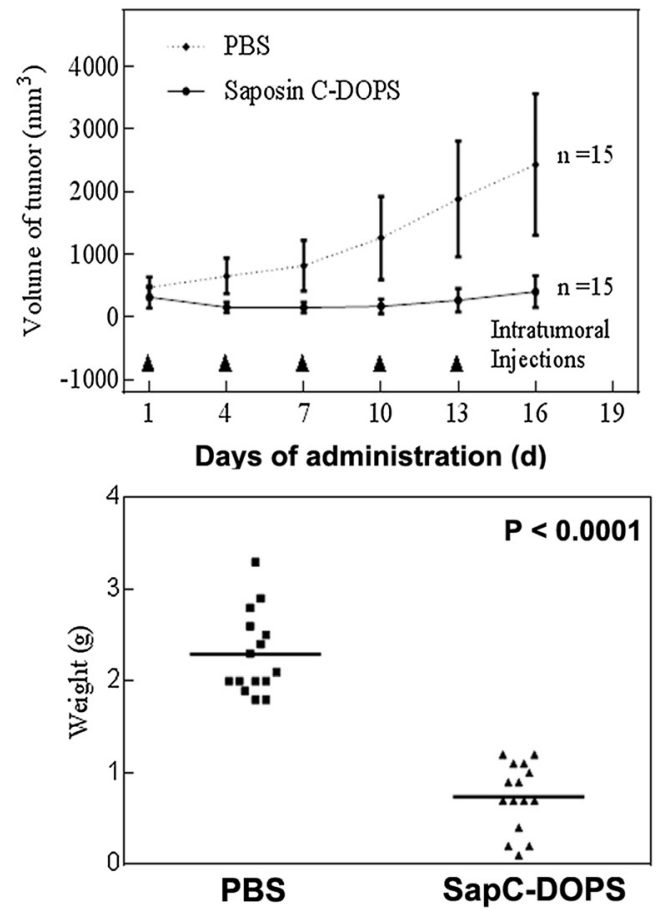

D
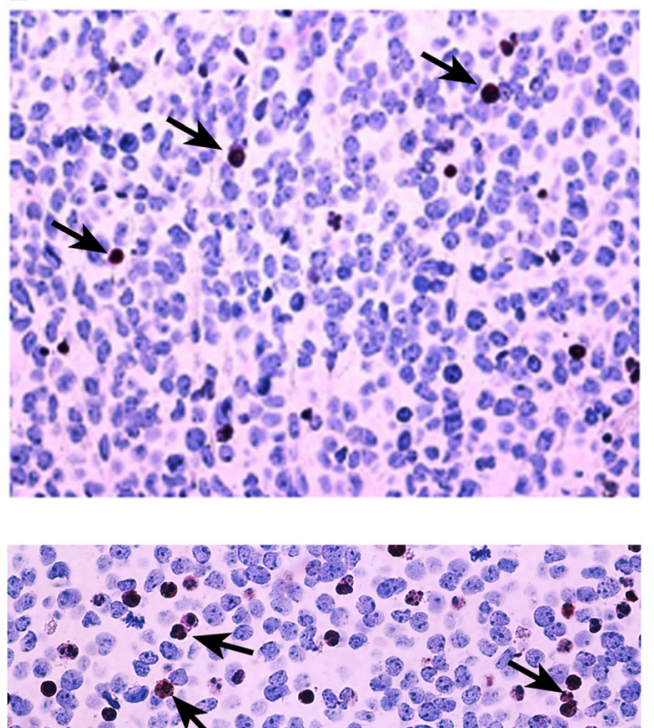

SapC-DOPS

Figure 1 (See legend on next page.) 
(See figure on previous page.)

Figure 1 Preparation and tumor-targeting potential of SapC-DOPS in mice xenografts. A) Treatment of athymic nude mice bearing neuroblastoma (CHLA-20) xenografts with CVM-labeled SapC-DOPS (1), CVM-DOPS (2) or SapC-CVM (3). Animals were imaged 24 h after injection with exposure time of $1 \mathrm{~s}$. Saposin C, $4.2 \mathrm{mg} / \mathrm{kg}$; dioleoylphosphatidylserine, $2 \mathrm{mg} / \mathrm{kg}$; CellVue Maroon, 6 umol. B) Evaluation of tumor burden in SapC-DOPS treated mice xenografts. Mice xenografts $(n=15)$ were treated with five intratumoral injections of SapC-DOPS (SapC 4 mg/kg, DOPS $2 \mathrm{mg} / \mathrm{kg}$ ) or PBS every 3 days and followed for tumor growth. C) Histological examination of the neuroblastoma tumor tissue in mice after intratumoral injection of DOPS and SapC-DOPS. Original magnification: 1000x. D) Evaluation of apoptosis by TUNEL staining (arrows) in DOPS- and SapC-DOPS treated tumors. Original magnification: 40x.

levels of sphinganine, sphingosine and ceramides i.e. molecules capable of regulating mitochondrial function were reported earlier [8] the role of SapC-DOPS in mitochondrial function was not critically evaluated. Viability assays in cultured cells measured after $72 \mathrm{~h}$, showed that SapC-DOPS induced significant cell death in a dose-dependent manner at a concentration of $15 \mu \mathrm{M}$ or above in SK-N-SH and IMR-32 human neuroblastoma cells, but not in normal, human Schwann cells $[P<0.001$; Figure 2A]. In contrast, the maximum loss of viability in Schwann cells when exposed to the highest concentration of $200 \mu \mathrm{M}$ SapC-DOPS was only around $10-15 \%$. IMR-32 cells proved to be more resistant to SapC-DOPS than SK-N-SH cells, in as much as $200 \mu \mathrm{M}$ dose did not induce further cell death, after a 3 day culture period. The microscopic changes observed were characteristic of apoptosis and included irregular shapes, nuclear chromatin condensation and cell shrinkage [Figure 2B, see arrows]. Treatment with up to $350 \mu \mathrm{M}$ DOPS alone did not elicit cell death [Additional file 1: Figure S1A]. Likewise, SK-N-SH and IMR-32 cells treated with SapC did not show any significant decrease in viability (data not

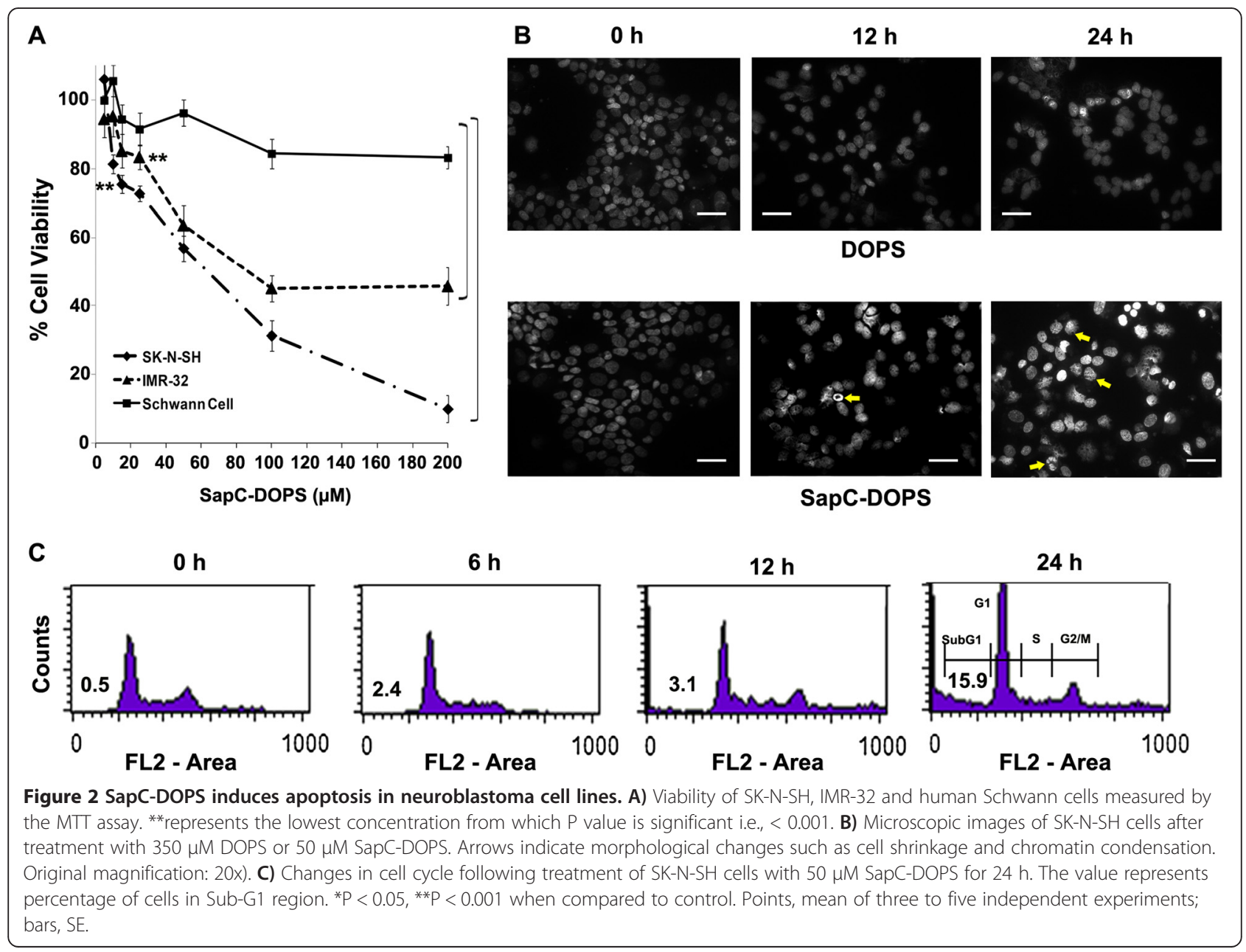


shown). Cell cycle analysis by flow cytometry showed substantial DNA fragmentation as indicated by the timedependent increase in sub- $\mathrm{G}_{0} / \mathrm{G} 1$ events [Figure $2 \mathrm{C}$ ]. A G6PD release assay, used to estimate necrotic cell death, yielded negative results $(\mathrm{P}=0.46 ; \mathrm{n}=5$; Additional file 1 : Figure S1B) and further confirmed that apoptosis is the predominant mechanism underlying SapC-DOPS-induced cell death.

\section{SapC-DOPS disrupts mitochondrial membrane potential in neuroblastoma cells}

Disruption of $\triangle \Psi M$, which indicates mitochondrial dysfunction and loss of membrane integrity, is considered an early step in apoptosis [31,32]. In healthy cells, the potentiometric mitochondrial dye JC-1 preferentially accumulates within the mitochondria and spontaneously aggregates into a polymer emitting red fluorescence. In apoptotic, unhealthy cells with low potential, JC-1 remains in monomeric form emitting green fluorescence. Treatment of neuroblastoma cells with SapC-DOPS resulted in pronounced loss of $\triangle \Psi M$ in both dose- and time-dependent manners [Figure 3A]. The decrease in red/green fluorescence ratio of $\mathrm{JC}-1$ was significant $(P<$ 0.001 ) after $4 \mathrm{~h}$ at a dose of $50 \mu \mathrm{M}$ SapC-DOPS, with a more pronounced effect at later times. At $24 \mathrm{~h}, 5 \mu \mathrm{M}$ was sufficient to significantly induce loss in $\triangle \Psi M(P<$ 0.01). Pretreatment with $50 \mu \mathrm{M}$ BA, a $\Delta \Psi \mathrm{M}$ stabilizer [33], prevented loss of $\triangle \Psi M$ [Figure $3 C$ ], and significantly attenuated SapC-DOPS induced cell death in SK$\mathrm{N}-\mathrm{SH}$ cells $[P<0.001$; Figure $3 \mathrm{D}]$. These observations suggest that a loss of $\Delta \Psi M$ underlies the induction of neuroblastoma apoptosis by SapC-DOPS.

SapC-DOPS induces translocation of apoptogenic proteins and oligomerization

During mitochondria-mediated apoptosis the outer mitochondrial membrane becomes permeable, a process that is necessary for apoptogenic protein release and caspase activation [34]. Smac, Cyto c, and AIF are proteins released to cytosol from mitochondria in response to death stimuli [35]; Smac and Cyto c cause apoptosis by caspase-dependent pathways whereas AIF works by caspase-independent pathways [36]. As shown in Figure 4A, treatment of SK-N-SH and IMR-32 cells with SapC-DOPS caused an increase in the expression of AIF, Smac and Cyto c. These changes were paralleled by a marked increase in the active caspase- 3 fragment, an early execution phase signal in human neuroblastoma [37], as well as in cleaved poly-ADP-ribose polymerase (cPARP), a substrate of caspase-3. Partial decreases in the expression levels of anti-apoptotic protein Bcl-2 were also observed, particularly in IMR-32 cells. Survivin, a member of the XIAP family of anti-apoptotic proteins, showed a transient increase at $6 \mathrm{~h}$ but returned near baseline levels at $24 \mathrm{~h}$ post-treatment in both cell lines. To determine whether SapC-DOPS causes a redistribution of Smac and Cyto c to the cytosol, mitochondrial as well as cytosolic levels of both proteins were estimated simultaneously by Western blotting. These results showed that Smac release preceded that of Cyto c (Figure 4B). Control experiments performed in SK-N-SH cells showed that DOPS alone did not alter the expression levels of apoptotic proteins [Additional file 2: Figure S2A]. Likewise, no change in apoptotic protein expression was noticed upon treatment of SK-N-SH and IMR-32 cells with SapC alone (data not shown). These results demonstrate that SapC-DOPS treatment increases AIF, Smac and Cyto c protein levels, triggers a cellular redistribution of Smac and Cyto c, and induces cPARP formation and activation of caspase- 3 .

\section{Bax activation is necessary for SapC-DOPS-induced apoptosis}

Bax, a crucial proapoptotic factor of the Bcl-2 family localizes to the cytosol but translocates to the mitochondria in response to various apoptotic stimuli [38]. Relocated Bax molecules facilitate mitochondrial release of Smac and/or Cyto $\mathrm{c}$ to the cytosol by forming channels on the outer mitochondrial membrane via homo- or hetero-dimerization with members of the permeability transition pore (PTP) such as the voltagedependent anionic channel (VDAC), the adenine nucleotide translocator, and cyclophylin D, among others[39]. In untreated SK-N-SH and IMR-32 cells, Bax proteins were predominantly found in the cytosolic fraction of [Figure 4C]. SapC-DOPS treatment induced Bax translocation from the cytosolic to the mitochondrial compartment [Figure 4C]. Bax polymerization, detected with a monoclonal antibody (6A7) that specifically recognizes Bax oligomers [27], was confirmed in whole cell extracts of SK-N-SH and IMR-32 cells [Figure 4D]. A sustained increase in total Bax monomer expression was detected $6 \mathrm{~h}$ after treatment in both cell lines.

\section{Relocation of Smac and Cyto $\mathrm{c}$ is independent of ROS formation, Cyclophilin D channel activity and increased $\mathrm{Ca}^{2+}$ levels}

Loss of $\triangle \Psi M$ results in several deleterious intracellular outcomes such as generation of ROS that promotes oxidative stress and the subsequent release of mitochondrial proteins Smac, Cyto c and AIF to the cytosol. Since generation of ROS has been shown to accelerate cell death in neuroblastoma cells [40], we examined the effects of SapC-DOPS on intracellular ROS formation. SapC-DOPS treatment induced significant mitochondrial superoxide formation, as shown by an increase in MitoSox-red fluorescence intensity, $24 \mathrm{~h}$ post-treatment, in both SK-N-SH and IMR-32 cells [Additional file 1: Figure S1C and S1D]. 


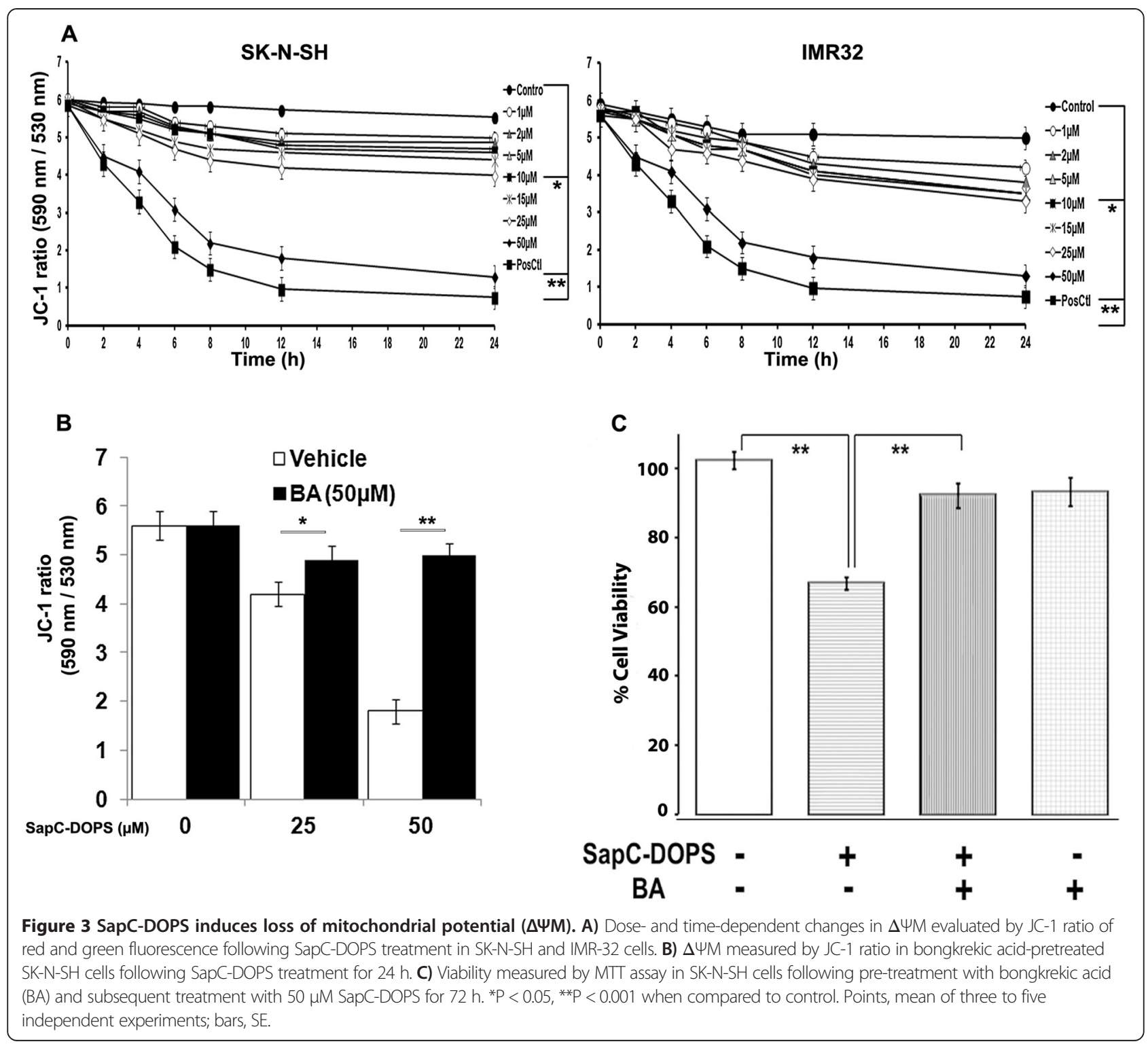

The next set of experiments was performed to differentiate whether apoptotic induction by SapC-DOPS is driven by ROS or the intrinsic pathway. First, we blocked ROS formation by pre-treating SK-N-SH cells with 2 mM NAC and evaluated Smac and Cyto c relocation. Pretreatment with NAC neither rescued cell viability (Figure 5A) nor prevented Smac and Cyto c relocation from mitochondria to cytosol (Figure 5B). These results suggest that induction of ROS does not mediate the apoptotic effects of SapCDOPS and may be a secondary consequence of SapCDOPS action.

The permeability transition pore (PTP), an incompletely characterized channel complex that may include Bax, is a major determinant of the mitochondrial release of Smac, Cyto c and AIF during apoptosis [41]. To determine the possible role of the PTP in SapC-DOPS mediated mobilization of Smac and Cyto c, SK-N-SH cells were pre-incubated with $1 \mu \mathrm{M}$ cyclosporine A, which blocks PTP opening by binding to cyclophilin D [42] As shown in Figure 5C, PTP inhibition did not alter the time-dependent relocation of Cyto c and Smac from mitochondria to the cytosol. Elevation of intracellular $\mathrm{Ca}^{2+}$ is an important trigger for PTP opening and the subsequent release of apoptogenic proteins. Flow cytometric analysis with the $\mathrm{Ca}^{2+}$-sensitive dye Fluo-3 AM showed no changes in intracellular $\mathrm{Ca}^{2+}$ levels following SapC-DOPS treatment [Figure 5D]. These results show that SapC-DOPS induces mitochondrial Smac and Cyto c release without mediation by Cyclophilin D/PTP or $\mathrm{Ca}^{2+}$. 


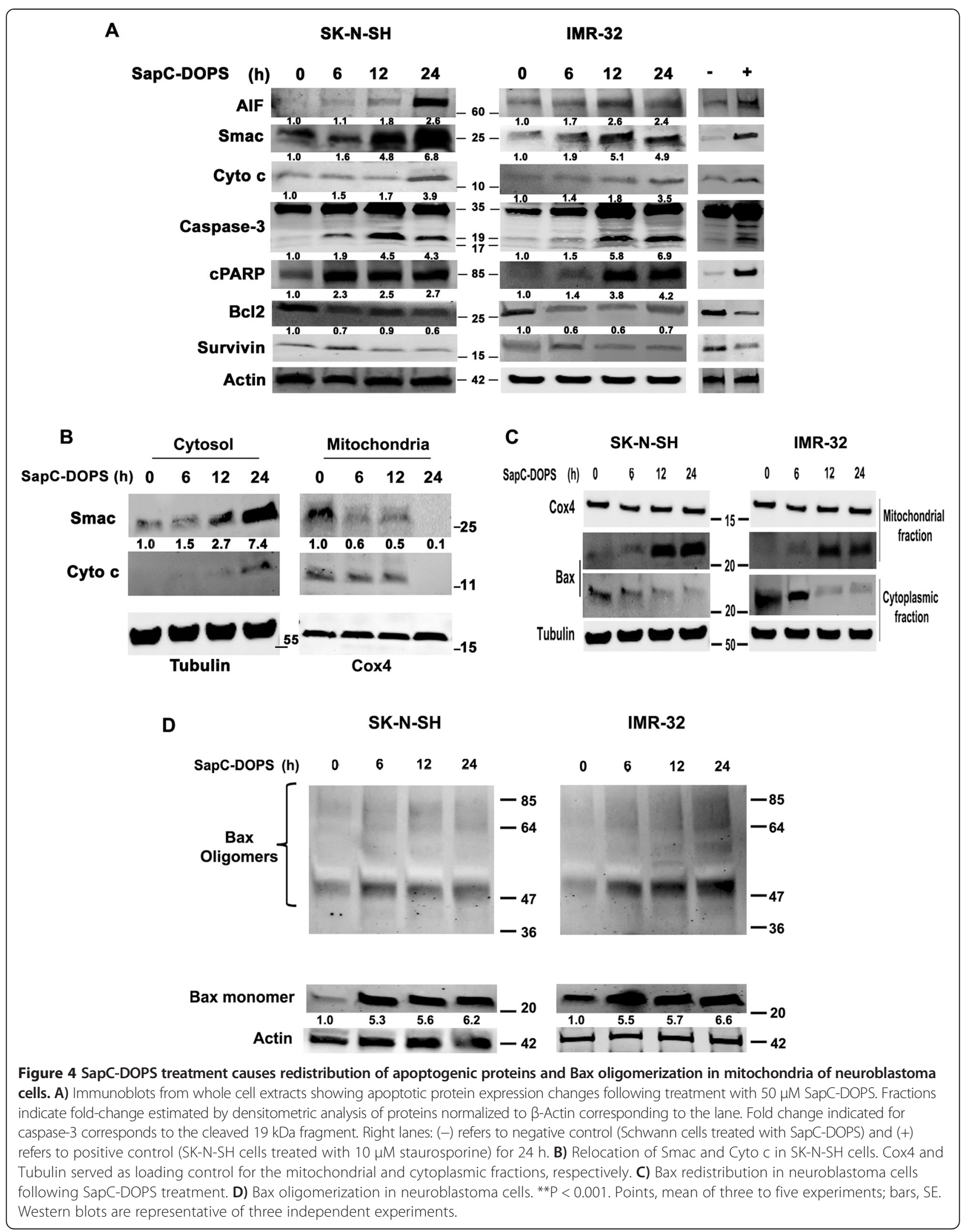




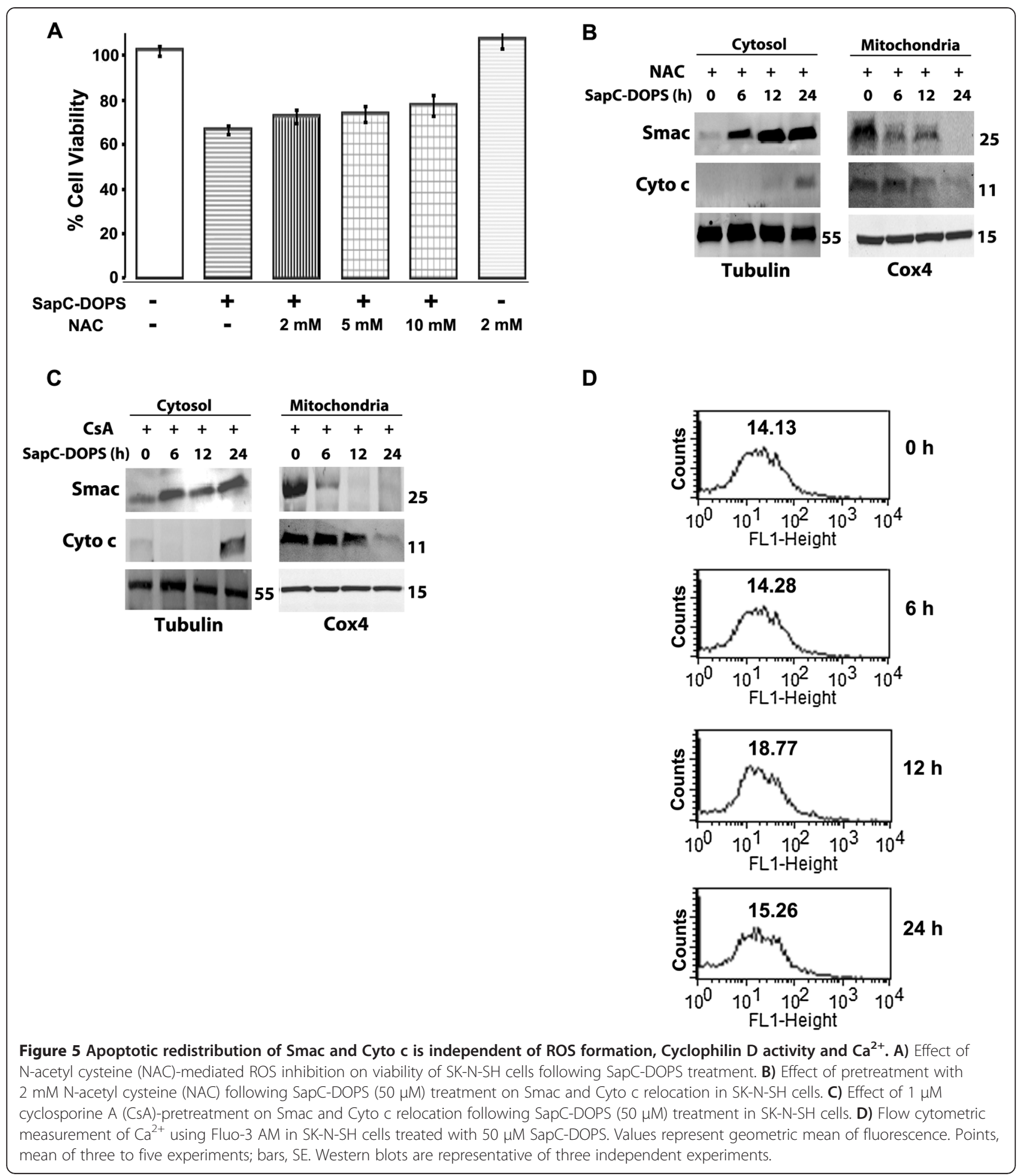

Smac and Bax play critical roles in SapC-DOPS induced apoptosis

Studies have shown that Smac/Diablo protein release can occur independently of Cyto c release from the mitochondria during apoptosis $[22,23]$. As noted above, Smac release occurred by $6 \mathrm{~h}$, the earliest time point in our study that coincided with caspase- 3 and PARP cleavage. Therefore, to assess the relevance of Smac in SapC-DOPS-induced apoptosis, we performed shRNA-mediated Smac knockdown in SK-N-SH cells. Cells were transfected with $50 \mathrm{nM}$ lentiviral 
Smac shRNA in vitro, and viable cell number assessed over time. Whole cell immunoblots confirmed significantly decreased Smac protein levels, as measured $48 \mathrm{~h}$ after transfection, with selective protein knockdown of $>96 \%$ [Additional file 2: Figure S2B]. Apoptosis-related off-target effects were ruled out by comparing levels of Cyto $\mathrm{c}$ in control and shSmac-treated cells. Knocking down Smac expression significantly decreased SapC-DOPS-induced apoptosis as denoted by reduced cell death $(P<0.001$; Figure $6 \mathrm{~A}$ ), and prevented the $\Delta \Psi \mathrm{M}$ loss, reflected by increased JC-1 red/green fluorescence ratio (Figure 6B). While Smac knockdown did not affect mitochondrial release of AIF and Cyto c, the translocation of Bax to mitochondria was abrogated [Figure $6 \mathrm{C}$ ] and the expression of cleaved caspase- 3 fragments was significantly attenuated in SapC-DOPS-treated SK-N-SH cells $[P<0.01$; Figure 6D]. Taken together, these findings suggest that Smac plays a critical functional role in SapC-DOPS-induced apoptosis.

Because SapC-DOPS induces Bax translocation and oligomerization, we questioned whether Bax is necessary for its pro-apoptotic action. SK-N-SH cells were treated with the Bax-inhibitory peptide V5 $(50 \mu \mathrm{M})$ or a control peptide $(50 \mu \mathrm{M})$ for 30 min followed by treatment with $50 \mu \mathrm{M}$ SapC-DOPS. Suggesting an important role for Bax in the mitochondrial depolarization caused by SapCDOPS, V5 pretreatment led to a significant reduction in cell death $[P<0.001$; Figure $7 \mathrm{~A}]$ and attenuation of $\Delta \Psi \mathrm{M}$ loss [Figure 7B]. Consistent with decreased apoptosis, Bax inhibition also attenuated Smac and Cyto c mitochondrial release and reduced caspase-3 activation [Figure 7C, 7D]. Pretreatment with the control peptide neither inhibited Bax nor decreased SapC-DOPS-induced apoptosis. These results indicate that Bax is a critical player during SapCDOPS induced apoptosis.

In summary, these findings strongly suggest that SapC-DOPS preferentially induces apoptosis in neuroblastoma by a mitochondrial-mediated pathway.

\section{Discussion}

This study shows that SapC-DOPS, an antitumor agent formed by the naturally-occurring protein Saposin C and DOPS, targets neuroblastoma cells and inhibits neuroblastoma growth in vitro and in vivo. Previous work from our lab has shown that the preferential targeting of SapC-DOPS nanovesicles to cancer cells, while sparing normal ones, is due to higher levels of exposed phosphatidylserine on their outer membranes [1,6-10]. Upon cell binding, SapC-DOPS is internalized and SapC activates lysosomal hydrolases that degrade glucosylceramide and sphingomyelin, resulting in the accumulation of ceramide [8], a well-known apoptosis inducer [18]. In the present study we perform a detailed analysis of SapC-DOPS actions in two neuroblastoma cell lines, and reveal that tumor toxicity results from mitochondrial- mediated apoptosis triggered by disrupted $\Delta \Psi \mathrm{M}$, mitochondrial release of Cyto $\mathrm{c}$ and Smac, Bax relocation and oligomerization, and activation of Caspase 3. With the efficacy of SapC-DOPS having been confirmed in numerous solid tumor models [1,6,8-10], the elucidation of SapC-DOPS mode of action is of critical importance to design clinical trials, predict clinical outcomes as well as anticipate and manage potential adverse effects.

Our in vitro results show that SapC-DOPS exerts dosedependent cytotoxicity in IMR-32 and SK-N-SH neuroblastoma cells, but have little effect on the viability of normal Schwann cells. Treated neuroblastoma cell cultures showed microscopy features typical of apoptosis, including cell shrinkage and chromatin condensation, while flow cytometric analysis of DNA content showed progressive DNA fragmentation. On the other hand, necrotic cell death was ruled out, as evidenced by a G6PD assay. Next, we addressed the molecular bases of SapC-DOPS induced apoptosis, by first evaluating possible changes in $\Delta \Psi M$. Mitochondria maintain $\triangle \Psi M$ by controlling ion transport via channels residing in the inner and outer mitochondrial membranes. Loss of $\triangle \Psi M$ is an early requirement of apoptosis [43] and precedes chromatin condensation [44]. Upon induction of apoptosis, a series of events induce mitochondrial outer membrane permeabilization (MOMP), altering $\triangle \Psi M$. Under certain conditions loss of $\triangle \Psi M$ acts as an initiator, whereas in others it follows its onset [45]. MOMP is mainly controlled by the Bcl-2 family of proteins that either reside on the mitochondrial membrane, or reassemble there after translocation from cytoplasm. Upon oligomerization, they form new channels that release mitochondrial apoptogenic proteins like Cyto c, Smac and AIF. We show here that SapC-DOPS nanovesicles induce, within $6 \mathrm{~h}$, a significant decrease in $\Delta \Psi \mathrm{M}$, which is paralleled by a decrease in mitochondrial Smac and increased cPARP and caspase 3 cleavage, denoting apoptotic cell death. Loss of $\triangle \Psi M$ is critical for SapC-DOPS tumor toxicity, as cell viability was significantly rescued following pre-treatment of cells with the $\triangle \Psi M$ stabilizer agent BA. Further experiments showed that SapC-DOPS induced a delayed release (by $24 \mathrm{~h}$ ) of other important pro-apoptotic proteins, namely Cyto $\mathrm{c}$ and AIF, as well as translocation of Bax from cytosol to mitochondria with oligomerization of Bax monomers.

The nature and specificity of mitochondrial protein channels and their individual preferences towards the apoptogenic proteins Smac and Cyto $\mathrm{c}$ is under intense debate [46]. However, accumulating evidence suggests that several channels function independently or in conjunction with the $\mathrm{Bcl}-2$ family to determine the internal milieu of the organelle $[41,47]$. The phenomenon termed "mitochondrial permeability transition" (MPT) reflects the opening of the mitochondrial permeability transition pore (PTP), triggering an influx of water into 


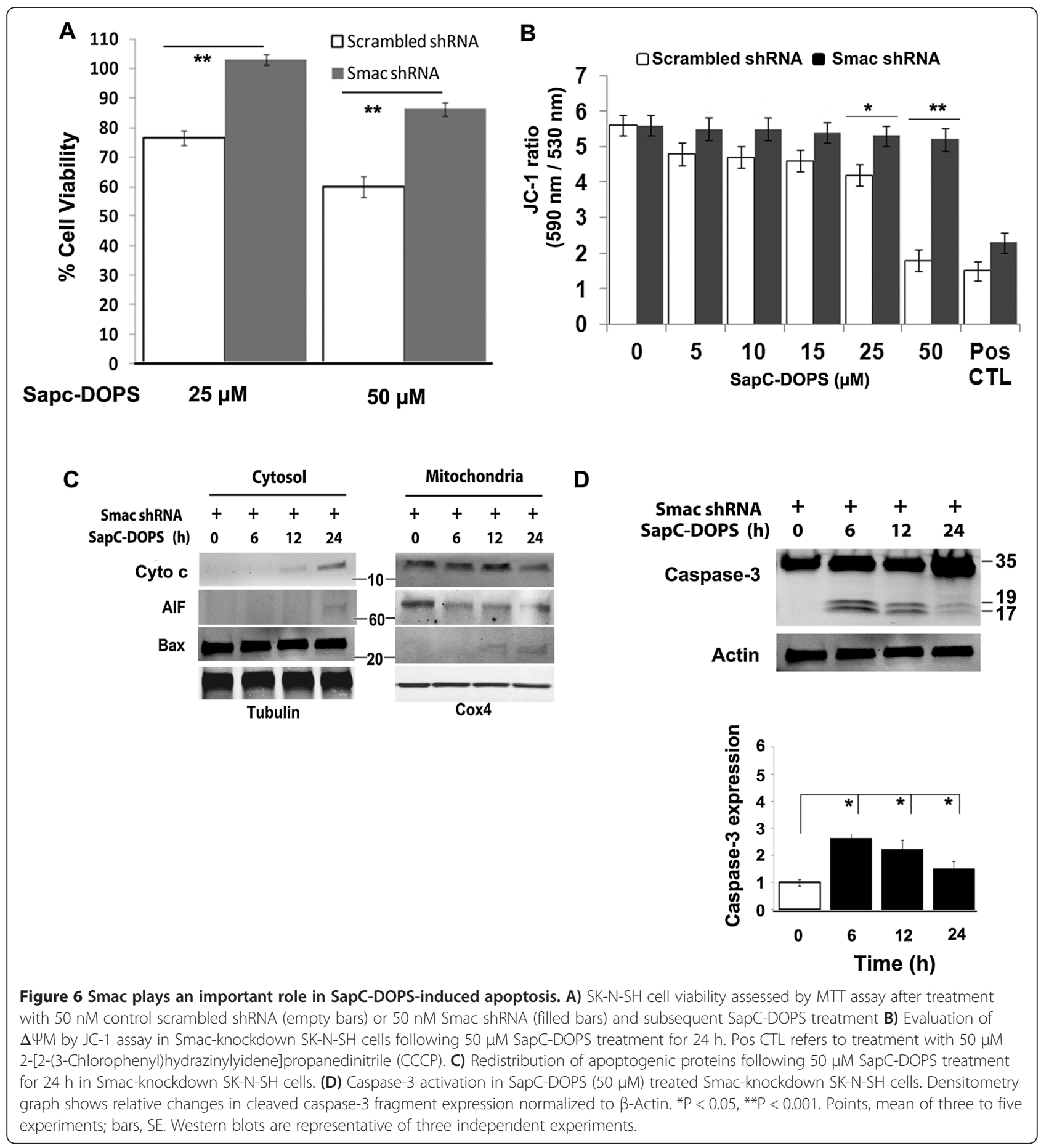

the mitochondrial matrix due to osmosis and resulting in MOMP, which promotes apoptotic caspase-dependent and -independent cell death. VDAC, ANT, Cyclophilin $\mathrm{D}$ and the Translocator protein $(18 \mathrm{kD})$ are putative constituents of the PTP [47,48], although knockout experiments have shown VDAC and ANT to be dispensable for MPT-driven MOMP, suggesting that alternate channels may regulate cell death [48]. Pre-treatment with cyclosporine A, which inhibits the PTP by binding to Cyclophilin D, failed to prevent SapC-DOPS-induced mitochondrial efflux of Smac or Cyto c. Mitochondrial $\mathrm{Ca}^{2+}$ overload is a critical activator of the PTP [49]. In this study, however, flow cytometry with the $\mathrm{Ca}^{2+}$-sensitive dye Fluo-3 AM showed that total intracellular $\mathrm{Ca}^{2+}$ 


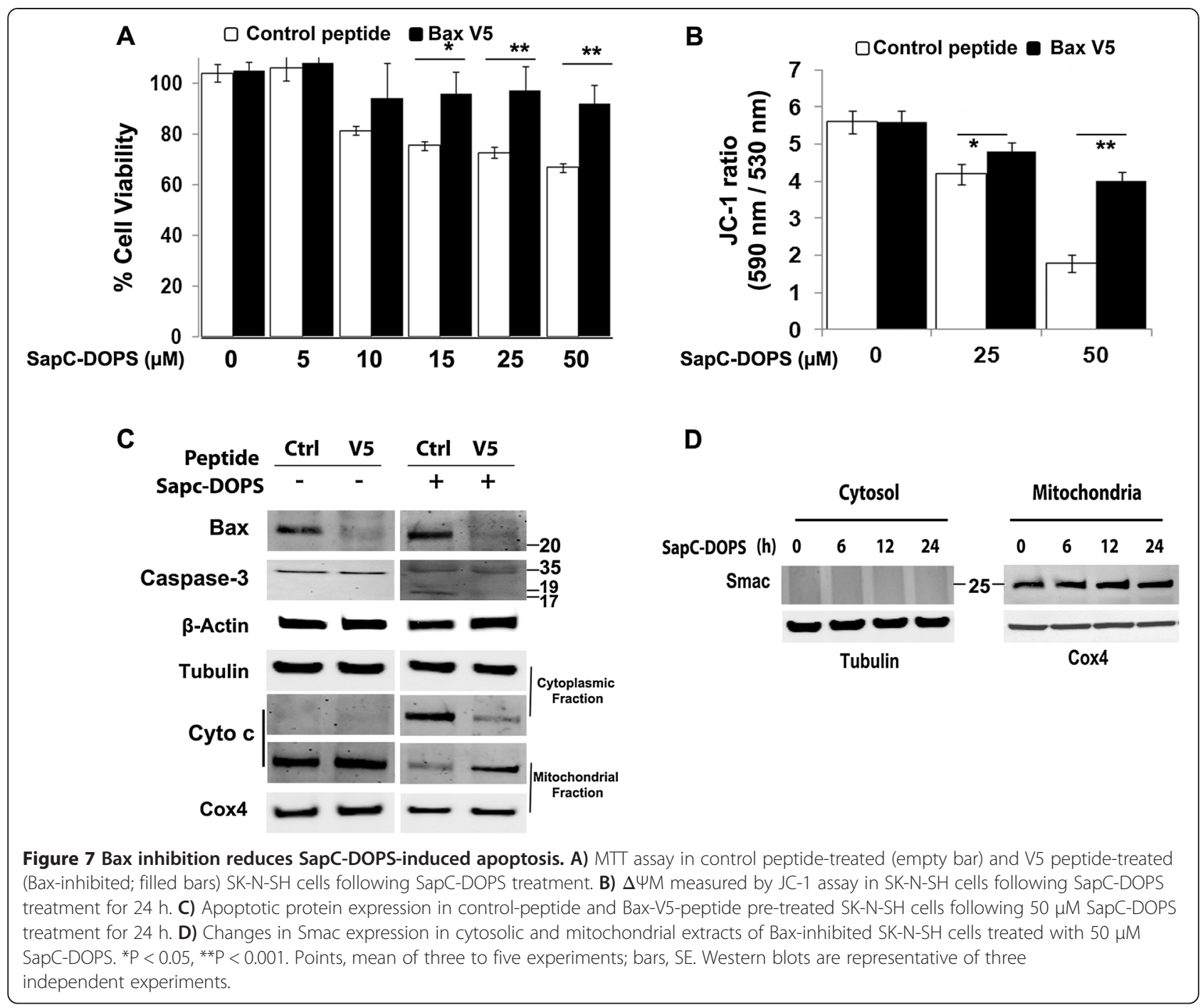

levels were not significantly altered after SapC-DOPS treatment. Collectively these results indicate that neither Cyclophilin D-mediated PTP opening nor intracellular $\mathrm{Ca}^{2+}$ elevations are critical for SapC-DOPS-induced apoptosis of neuroblastoma cells.

Bax oligomerization is proposed to form megachannels in the outer mitochondrial membrane facilitating proapoptotic protein release [36]. In support of an essential role of Bax, we observed that mitochondrial Bax translocation is rapid following SapC-DOPS treatment, and is necessary for Smac and Cyto c release from the mitochondria. However, significant oligomerization of Bax is only noticed at $24 \mathrm{~h}$, which coincides with the peak of Smac and Cyto c release from the mitochondria. Although many studies suggest that in the absence of Bax no cytosolic Smac release occurs [50], there are reports which show that cytotoxins [51] as well as apoptosis inducers such as AT-101 [27] directly target mitochondria and trigger Smac release irrespective of Bax activation. In the present study, Bax inhibition led to complete loss of cytosolic Smac release and an attenuated apoptosis as seen by diminished Cyto c release and caspase- 3 activation. These results indicate that Bax is required for SapC-DOPS induced apoptosis. However, the trigger for Bax polymerization is currently unknown. ROS formation may trigger Bax conformational change and translocation [52]. Consistent with this, we observed an increase in ROS formation by $6 \mathrm{~h}$ after SapC-DOPS treatment. However, generation of ROS is not required for apoptosis induction since pretreatment with ROS scavenger, $\mathrm{N}$-acetyl cysteine (NAC) failed to prevent SapC-DOPS-induced cytosolic Smac and Cyto c release. Therefore, our results imply that SapC-DOPS-induced ROS formation is not critical for apoptosis, but may enhance Bax oligomerization to form megachannels. The relationship between Smac release and Bax activation is 
complex and is still under active investigation. Early mitochondrial release pointed to a crucial role for Smac in SapC-DOPS induced apoptosis. It has been reported that initial Smac release requires active caspases [53], and we observed caspase activation corresponding to this time point. In general, during apoptosis the temporal release of Cyto c from mitochondria precedes Smac release [54]. However, some anticancer drugs selectively release Smac rather than Cyto c [55]. In agreement with the latter, we noticed selective cytosolic Smac release at the early stages of SapC-DOPS-induced apoptosis, whereas Cyto c release was not evident until $24 \mathrm{~h}$. A marked increase in cell viability, retention of $\triangle \Psi M$ and reduction in SapC-DOPS induced apoptosis as seen by a decrease in cytosolic AIF and Cyto c release in Smac knockout cells further confirmed the essential role of Smac. These results are consistent with other reports that show Smac as an essential pro-apoptotic molecule that determines anticancer activity [56].

The two human neuroblastoma cell lines we used in the present study have been shown to differ in their expression of MYCN protein, which plays an important role in the synergistic activation of Smac in some neuroblastoma cells [57]. However, we observed that SapC-DOPS induced mitochondrial Smac and Cyto c release pattern was similar in the two neuroblastoma cell lines examined, despite different MYCN status. Further studies may be needed to clarify if SapC-DOPS-induced Bax oligomerization culminates in Smac release independently, or in combination with other Bcl-2 family members.

In summary, our present findings indicate that SapCDOPS shows selective in vivo tumor targeting and exert significant tumor inhibition in mice bearing human neuroblastoma xenografts. Apoptosis was observed in vivo and in vitro and occurred through a mitochondrial pathway as demonstrated by a loss of mitochondrial $\triangle \Psi M$ and increased mitochondrial superoxide formation, with mitochondrial release of apoptogenic proteins such as AIF, Smac and Cyto c, and mitochondrial translocation and polymerization of cytosolic Bax. Gene knockdown and inhibition studies established Smac and Bax as major regulators of SapC-DOPS-induced apoptosis of neuroblastoma cells. These results, and the benign safety profile evidenced by several studies, suggest that SapC-DOPS may provide an effective therapeutic approach against neuroblastoma.

\section{Additional files}

Additional file 1: Figure S1. Evaluation of necrosis and mitochondrial superoxide formation. A) MTT assay of SK-N-SH cells treated with DOPS. B) Necrosis measured by G6PD release in SK-N-SH cells following treatment with SapC, DOPS and SapC-DOPS for 24 h. C) Mean MitoSox-Red fluorescence following $50 \mu \mathrm{M}$ SapC-DOPS treatment of neuroblastoma cells for $24 \mathrm{~h}$. Pos CTL stands for pre-treatment with $20 \mu \mathrm{M}$ Antimycin A. D) Quantification of fold changes in MitoSox intensity after treatment with $50 \mu \mathrm{M}$ SapC, $350 \mu \mathrm{M}$ DOPS or SapC-DOPS $(25,50 \mu \mathrm{M})$ for $24 \mathrm{~h}$.
Additional file 2: Figure S2. Protein expression analysis in SK-N-SH cells. A) Expression of apoptotic proteins following treatment with $350 \mu \mathrm{M}$ DOPS. Fractions indicate fold-change estimated by densitometric analysis of proteins normalized to $\beta$-Actin corresponding to the lane. B) ShRNA-mediated knockdown of Smac in SK-N-SH cells. Percentages represent reduction in Smac normalized to $\beta$-Actin.

\section{Competing interests}

Patents are pending for the intellectual property disclosed in this manuscript. $X$. Qi is listed as an inventor on the patent for SapC-DOPS technology that is the subject of this research. Consistent with current Cincinnati Children's Hospital Medical Center policies, the development and commercialization of this technology has been licensed to Bexion Pharmaceuticals, LLC, in which X. Qi, holds a minor $(<5 \%)$ equity interest. The other authors declared no conflict of interest.

\section{Authors' contributions}

Conceived and designed the experiments: SMK, ZC, RSF, XQ. Performed the experiments: SMK, ZC, XQ. Analyzed the data: SMK, ZC, VMB, SDV, RSF, XQ. Contributed reagents/materials/analysis tools: SMK, ZC, RSF, XQ. Wrote the paper: SMK, XQ. Edited and approved the manuscript: SMK, ZC, VMB, SDV, RSF, XQ.

\section{Acknowledgements}

The authors thank the George Thomas lab (The Vontz Center for Molecular Studies, University of Cincinnati) for their assistance with Western blotting and imaging studies; and Dr. Harold Davis, for comments on the manuscript.

\section{Funding information}

This work was supported in part by 1R01CA158372 (to X. Qi), Research Funds and Hematology-Oncology Programmatic Support from University of Cincinnati College of Medicine (to X. Qi), and New Drug State Key Project Grant Number 009ZX09102-205 (to X. Qi).

Received: 6 June 2014 Accepted: 9 March 2015

Published online: 08 April 2015

\section{References}

1. Blanco VM, Chu Z, Vallabhapurapu SD, Sulaiman MK, Kendler A, Rixe O, et al. Phosphatidylserine-selective targeting and anticancer effects of SapC-DOPS nanovesicles on brain tumors. Oncotarget. 2014;5:7105-18.

2. Farrell D, Ptak K, Panaro NJ, Grodzinski P. Nanotechnology-based cancer therapeutics-promise and challenge-lessons learned through the $\mathrm{NCl}$ Alliance for Nanotechnology in Cancer. Pharm Res. 2011;28:273-8.

3. Olowokure O, Qi X. Pancreatic cancer: current standards, working towards a new therapeutic approach. Expert Rev Anticancer Ther. 2014;14:495-7.

4. van Vlerken LE, Vyas TK, Amiji MM. Poly(ethylene glycol)-modified nanocarriers for tumor-targeted and intracellular delivery. Pharm Res. 2007;24:1405-14

5. Chu Z, LaSance K, Blanco V, Kwon CH, Kaur B, Frederick M, et al. In Vivo Optical Imaging of Brain Tumors and Arthritis Using Fluorescent SapC-DOPS Nanovesicles. J Vis Exp. 2014;87:e51187. 1-7.

6. Chu Z, Abu-Baker S, Palascak MB, Ahmad SA, Franco RS, Qi X. Targeting and cytotoxicity of SapC-DOPS nanovesicles in pancreatic cancer. PLoS One. 2013:8:e75507.

7. Kaimal V, Chu Z, Mahller YY, Papahadjopoulos-Sternberg B, Cripe TP, Holland SK, et al. Saposin C coupled lipid nanovesicles enable cancer-selective optical and magnetic resonance imaging. Mol Imaging Biol. 2011;13:886-97.

8. Qi X, Chu Z, Mahller YY, Stringer KF, Witte DP, Cripe TP. Cancer-selective targeting and cytotoxicity by liposomal-coupled lysosomal saposin C protein. Clin Cancer Res. 2009;15:5840-51.

9. Abu-Baker S, Chu Z, Stevens A, Li J, Qi X. Cytotoxicity and selectivity in skin cancer by sapc-DOPS nanovesicles. J Cancer Ther. 2012;3:6.

10. Wojton J, Chu Z, Mathsyaraja H, Meisen WH, Denton N, Kwon CH, et al. Systemic delivery of SapC-DOPS has antiangiogenic and antitumor effects against glioblastoma. Mol Ther. 2013;21:1517-25.

11. Winter PM, Pearce J, Chu Z, McPherson CM, Takigiku R, Lee JH, Qi X: Imaging of brain tumors with paramagnetic vesicles targeted to phosphatidylserine. J Magn Reson Imaging 2014;41:1079-1087.

12. Zhao S, Chu Z, Blanco VM, Nie Y, Hou Y, Qi X. SapC-DOPS nanovesicles as targeted therapy for lung cancer. Mol Cancer Ther. 2015;14:491-8. 
13. Qi X, Chu Z. Fusogenic domain and lysines in saposin C. Arch Biochem Biophys. 2004;424:210-8.

14. Liu A, Qi X. Molecular dynamics simulation of saposin C-membrane binding. Open Struct Biol J. 2008;2:21-30.

15. Brodeur GM. Neuroblastoma: biological insights into a clinical enigma. Nat Rev Cancer. 2003;3:203-16.

16. Berthold F, Boos J, Burdach S, Erttmann R, Henze G, Hermann J, et al. Myeloablative megatherapy with autologous stem-cell rescue versus oral maintenance chemotherapy as consolidation treatment in patients with high-risk neuroblastoma: a randomised controlled trial. Lancet Oncol. 2005;6:649-58.

17. Laverdiere C, Cheung NK, Kushner BH, Kramer K, Modak S, LaQuaglia MP, et al. Long-term complications in survivors of advanced stage neuroblastoma. Pediatr Blood Cancer. 2005:45:324-32.

18. von Haefen C, Wieder T, Gillissen B, Starck L, Graupner V, Dorken B, et al. Ceramide induces mitochondrial activation and apoptosis via a Baxdependent pathway in human carcinoma cells. Oncogene. 2002;21:4009-19.

19. Desagher S, Martinou JC. Mitochondria as the central control point of apoptosis. Trends Cell Biol. 2000;10:369-77.

20. Phillips DC, Martin S, Doyle BT, Houghton JA. Sphingosine-induced apoptosis in rhabdomyosarcoma cell lines is dependent on pre-mitochondrial Bax activation and post-mitochondrial caspases. Cancer Res. 2007;67:756-64.

21. Asakura T, Ohkawa K. Chemotherapeutic agents that induce mitochondrial apoptosis. Curr Cancer Drug Targets. 2004;4:577-90.

22. Du C, Fang M, Li Y, Li L, Wang X. Smac, a mitochondrial protein that promotes cytochrome c-dependent caspase activation by eliminating IAP inhibition. Cell. 2000;102:33-42.

23. Verhagen AM, Ekert PG, Pakusch M, Silke J, Connolly LM, Reid GE, et al. Identification of DIABLO, a mammalian protein that promotes apoptosis by binding to and antagonizing IAP proteins. Cell. 2000;102:43-53.

24. Gogvadze V, Orrenius S, Zhivotovsky B. Mitochondria as targets for cancer chemotherapy. Semin Cancer Biol. 2009:19:57-66.

25. Nieh MP, Pencer J, Katsaras J, Qi X. Spontaneously forming ellipsoidal phospholipid unilamellar vesicles and their interactions with helical domains of saposin C. Langmuir. 2006;22:11028-33.

26. Keshelava N, Seeger RC, Groshen S, Reynolds CP. Drug resistance patterns of human neuroblastoma cell lines derived from patients at different phases of therapy. Cancer Res. 1998;58:5396-405.

27. Hu W, Wang F, Tang J, Liu X, Yuan Z, Nie C, et al. Proapoptotic protein Smac mediates apoptosis in cisplatin-resistant ovarian cancer cells when treated with the anti-tumor agent AT101. J Biol Chem. 2012;287:68-80.

28. Lee JH, Choi SH, Baek MW, Kim MH, Kim HJ, Kim SH, et al. CoCl2 induces apoptosis through the mitochondria- and death receptor-mediated pathway in the mouse embryonic stem cells. Mol Cell Biochem. 2013;379:133-40.

29. Yu K, He Y, Yeung LW, Lam PK, Wu RS, Zhou B. DE-71-induced apoptosis involving intracellular calcium and the Bax-mitochondria-caspase protease pathway in human neuroblastoma cells in vitro. Toxicol Sci. 2008:104:341-51.

30. Ferraz da Costa DC, Casanova FA, Quarti J, Malheiros MS, Sanches D, Dos Santos PS, Fialho E, Silva JL: Transient transfection of a wild-type p53 gene triggers resveratrol-induced apoptosis in cancer cells. PLoS One 2012, 7:e48746.

31. Ly JD, Grubb DR, Lawen A. The mitochondrial membrane potential (deltapsi (m)) in apoptosis; an update. Apoptosis. 2003:8:115-28.

32. Trachootham D, Zhou Y, Zhang H, Demizu Y, Chen Z, Pelicano $H$, et al Selective killing of oncogenically transformed cells through a ROS-mediated mechanism by beta-phenylethyl isothiocyanate. Cancer Cell. 2006;10:241-52.

33. Zamzami N, Marchetti P, Castedo M, Hirsch T, Susin SA, Masse B, et al. Inhibitors of permeability transition interfere with the disruption of the mitochondrial transmembrane potential during apoptosis. FEBS Lett. 1996:384:53-7.

34. Yang J, Liu X, Bhalla K, Kim CN, Ibrado AM, Cai J, et al. Prevention of apoptosis by $\mathrm{BCl}-2$ : release of cytochrome $\mathrm{c}$ from mitochondria blocked. Science. 1997;275:1129-32.

35. Dejean LM, Ryu SY, Martinez-Caballero S, Teijido O, Peixoto PM, Kinnally KW. MAC and Bcl-2 family proteins conspire in a deadly plot. Biochim Biophys Acta. 2010;1797:1231-8.

36. Wang $X$. The expanding role of mitochondria in apoptosis. Genes Dev. 2001;15:2922-33.

37. Bursztajn S, Feng JJ, Berman SA, Nanda A. Poly (ADP-ribose) polymerase induction is an early signal of apoptosis in human neuroblastoma. Brain Res Mol Brain Res. 2000;76:363-76.
38. Karbowski M, Lee YJ, Gaume B, Jeong SY, Frank S, Nechushtan A, et al. Spatial and temporal association of Bax with mitochondrial fission sites, Drp1, and Mfn2 during apoptosis. J Cell Biol. 2002;159:931-8.

39. Borner $\mathrm{C}$. The $\mathrm{BCl}-2$ protein family: sensors and checkpoints for life-or-death decisions. Mol Immunol. 2003;39:615-47.

40. Veas-Perez de Tudela M, Delgado-Esteban M, Cuende J, Bolanos JP, Almeida A: Human neuroblastoma cells with MYCN amplification are selectively resistant to oxidative stress by transcriptionally up-regulating glutamate cysteine ligase. J Neurochem 2010, 113:819-825.

41. Kroemer G, Galluzzi L, Brenner C. Mitochondrial membrane permeabilization in cell death. Physiol Rev. 2007:87:99-163.

42. Baines CP, Kaiser RA, Purcell NH, Blair NS, Osinska H, Hambleton MA, et al. Loss of cyclophilin D reveals a critical role for mitochondrial permeability transition in cell death. Nature. 2005;434:658-62.

43. Barbu A, Welsh N, Saldeen J. Cytokine-induced apoptosis and necrosis are preceded by disruption of the mitochondrial membrane potential (Deltapsi(m)) in pancreatic RINm5F cells: prevention by Bcl-2. Mol Cell Endocrinol. 2002;190:75-82.

44. Vieira HL, Boya P, Cohen I, El Hamel C, Haouzi D, Druillenec S, et al. Cell permeable $\mathrm{BH} 3$-peptides overcome the cytoprotective effect of $\mathrm{BCl}-2$ and BCl-X(L). Oncogene. 2002;21:1963-77.

45. Finucane DM, Waterhouse NJ, Amarante-Mendes GP, Cotter TG, Green DR. Collapse of the inner mitochondrial transmembrane potential is not required for apoptosis of HL60 cells. Exp Cell Res. 1999:251:166-74.

46. Renault TT, Chipuk JE. Death upon a kiss: mitochondrial outer membrane composition and organelle communication govern sensitivity to BAK/BAXdependent apoptosis. Chem Biol. 2014;21:114-23.

47. Alavian KN, Beutner G, Lazrove E, Sacchetti S, Park HA, Licznerski P, et al. An uncoupling channel within the c-subunit ring of the F1FO ATP synthase is the mitochondrial permeability transition pore. Proc Natl Acad Sci U S A. 2014:111:10580-5.

48. Bonora M, Bononi A, De Marchi E, Giorgi C, Lebiedzinska M, Marchi S, et al. Role of the c subunit of the FO ATP synthase in mitochondrial permeability transition. Cell Cycle. 2013;12:674-83.

49. Bernardi P, Vassanelli S, Veronese P, Colonna R, Szabo I, Zoratti M. Modulation of the mitochondrial permeability transition pore. Effect of protons and divalent cations. J Biol Chem. 1992;267:2934-9.

50. Kohli M, Yu J, Seaman C, Bardelli A, Kinzler KW, Vogelstein B, et al. SMAC/ Diablo-dependent apoptosis induced by nonsteroidal antiinflammatory drugs (NSAIDs) in colon cancer cells. Proc Natl Acad Sci U S A. 2004;101:16897-902

51. Genestier AL, Michallet MC, Prevost G, Bellot G, Chalabreysse L, Peyrol S, et al. Staphylococcus aureus Panton-Valentine leukocidin directly targets mitochondria and induces Bax-independent apoptosis of human neutrophils. J Clin Invest. 2005;115:3117-27.

52. Zheng $Y$, Yamaguchi $H$, Tian $C$, Lee MW, Tang $H$, Wang HG, et al. Arsenic trioxide $(\mathrm{As}(2) \mathrm{O}(3))$ induces apoptosis through activation of Bax in hematopoietic cells. Oncogene. 2005:24:3339-47.

53. Adrain C, Creagh EM, Martin SJ. Apoptosis-associated release of Smac/DIA$\mathrm{BLO}$ from mitochondria requires active caspases and is blocked by $\mathrm{BCl}-2$. EMBO J. 2001;20:6627-36.

54. Gao W, Pu Y, Luo KQ, Chang DC. Temporal relationship between cytochrome $\mathrm{c}$ release and mitochondrial swelling during UV-induced apoptosis in living HeLa cells. J Cell Sci. 2001;114:2855-62.

55. Rudy A, Lopez-Anton N, Barth N, Pettit GR, Dirsch VM, Schulze-Osthoff K et al. Role of Smac in cephalostatin-induced cell death. Cell Death Differ. 2008:15:1930-40.

56. Vaux DL, Silke J. IAPs, RINGs and ubiquitylation. Nat Rev Mol Cell Biol. 2005:6:287-97.

57. Cui H, Li T, Ding HF. Linking of N-Myc to death receptor machinery in neuroblastoma cells. J Biol Chem. 2005;280:9474-81. 